\title{
Fluctuating consciousness caused by hydrocephalus: A complication of aortic valve replacement
}

\author{
Martin H. Chamberlain, FRCS, and Chandi Ratnatunga, FRCS, Oxford, United Kingdom
}

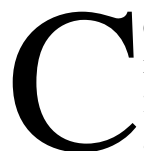

entral nervous system injury is a frequent and distressing complication of cardiopulmonary bypass. Up to $2.8 \%$ of patients undergoing simultaneous coronary artery bypass grafting and aortic valve replacement have a stroke. ${ }^{1}$ The majority of perioperative strokes are thought to be embolic, either from crossclamping of the aorta or from excision of heavily calcified aortic valves. Infratentorial strokes are the least frequent. ${ }^{2}$ After stroke in the posterior fossa, obstructive hydrocephalus develops in $10 \%$ to $25 \% .^{3}$ This may be due to infarction or hemorrhage. Edema leads to herniation and obstructive hydrocephalus, which is a life-threatening complication. We

From Oxford Heart Centre, John Radcliffe Hospital, Oxford, United Kingdom.

Received for publication Aug 22, 2001; accepted for publication Sept 9, 2001.

Address for reprints: Chandana P. Ratnatunga, FRCS, Oxford Heart Centre, John Radcliffe Hospital, Headley Way, Headington, Oxford, OX3 9DU United Kingdom.

J Thorac Cardiovasc Surg 2002;123:566-7

Copyright $\odot 2002$ by The American Association for Thoracic Surgery

$0022-5223 / 2002 \$ 35.00+0 \quad \mathbf{1 2 / 5 4 / 1 2 0 3 2 8}$

doi:10.1067/mtc.2002.120328 present a case of obstructive hydrocephalus after coronary artery surgery and aortic valve replacement.

\section{Clinical Summary}

A 60-year-old man underwent elective coronary artery bypass surgery and aortic valve replacement. Echocardiography demonstrated calcified aortic stenosis with a gradient of $90 \mathrm{~mm} \mathrm{Hg}$. Coronary angiography revealed 3 -vessel disease. His medical history was unremarkable with no previous neurologic events.

At operation, a 23-mm CarboMedics aortic valve prosthesis (Sulzer Carbomedics, Inc, Austin, Tex) was inserted with the use of routine cardiopulmonary bypass and blood cardioplegia. Three coronary artery bypass grafts were performed. Bypass and crossclamp times were 128 and 94 minutes, respectively. Thorough deairing was performed before removal of the aortic crossclamp. He was returned to the recovery unit and extubated that day. Altered speech was noted the following morning; however, the remainder of his neurologic examination was unremarkable and he was in sinus rhythm.

On the second postoperative day, while attempting to get up unaided, he fell, sustaining a frontal blow to the head. The Glasgow Coma Score (GCS) was 14 and neurologic observations were commenced. There were no focal neurologic abnormalities. Digoxin was commenced for atrial fibrillation. 
Over the next 72 hours the GCS fluctuated (12 to 15) and he was in intermittent atrial fibrillation. On day 5 he became unresponsive with a GCS of 5. Computed tomography (CT) demonstrated a partly hemorrhagic infarct in the cerebellar vermis and both cerebellar hemispheres (Figure 1, A). There was associated mass effect with effacement of the inferior part of the fourth ventricle, moderate hydrocephalus, and generalized sulcal effacement (Figure 1, B). No evidence of tonsillar descent was seen. The international normalized ratio was 3.2, which was corrected with fresh frozen plasma.

Neurosurgical referral was made and a temporary external ventricular drain was inserted with the aid of local anesthesia, draining clear cerebrospinal fluid and relieving the hydrocephalus. Anticoagulation was converted to intravenous heparin. The GCS returned to normal over the next 4 days, his condition improved, and the shunt was removed. Warfarin was recommenced and he was discharged to his home on day 20 after complete functional recovery.

\section{Comment}

Only one previous case of hydrocephalus following a cerebellar infarct after cardiopulmonary bypass has been reported in the literature. ${ }^{4}$ In our patient the most likely cause for the deterioration before the CT scan was the blow to the head after his fall. Progressive subdural hemorrhage (made worse by oral anticoagulation) was the expected finding on the CT scan, and not a cerebellar infarct.

A number of possible explanations exist for the development of the infarct. An intraoperative shower of emboli from aortic crossclamping and valve excision affecting the cerebellum and speech cortex simultaneously is one. An embolic event caused by atrial fibrillation is another. A third alternative is the development of a cerebellar infarct as a consequence of the blow to the head. Although rare, cerebellar infarcts resulting from blunt head trauma have been reported in the literature. ${ }^{5}$ The development of edema with or without hemorrhage in the cerebellar infarct resulted in occlusion of the canal of Sylvius and the progressive development of hydrocephalus.

The most likely sequence of events was an intraoperative cerebellar infarct caused by emboli. This resulted in altered speech and cerebellar ataxia precipitating a fall on the second postoperative day. The combination of blunt head trauma and developing hydrocephalus contributed to his fluctuating mental state and deterioration.

This case illustrates an unusual manifestation of a relatively common complication of cardiac surgery. When subtle fluctuations in levels of consciousness are present, the indications for neurologic opinion and investigations after cardiopulmonary bypass are much stronger. It demonstrates the value of CT in the diagnosis of hydrocephalus and indicates that early appropriate intervention can lead to a complete recovery.

\section{References}

1. Cernaianu AC, Teimouraz VV, Flum DR, Maurer M, Cilley JH Jr, Grosso MA, et al: Predictors of stroke after cardiac surgery. J Card Surg. 1995;10:334-9.

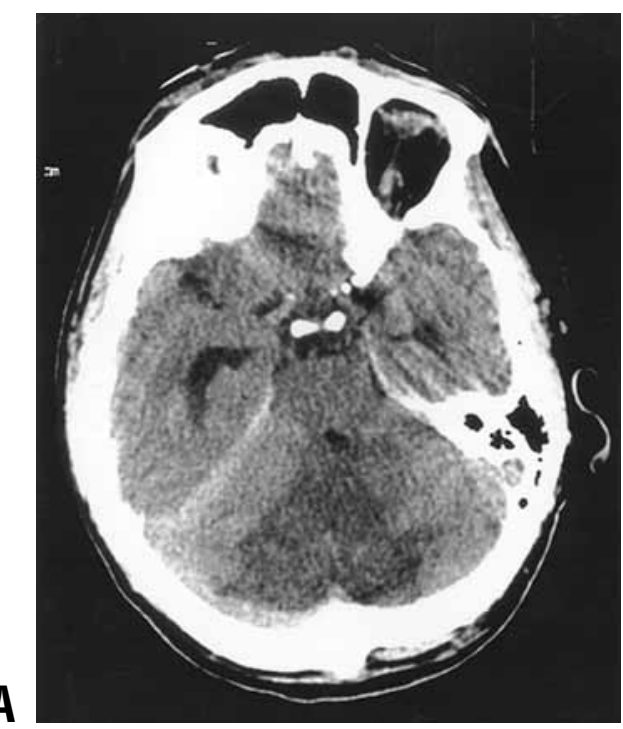

B

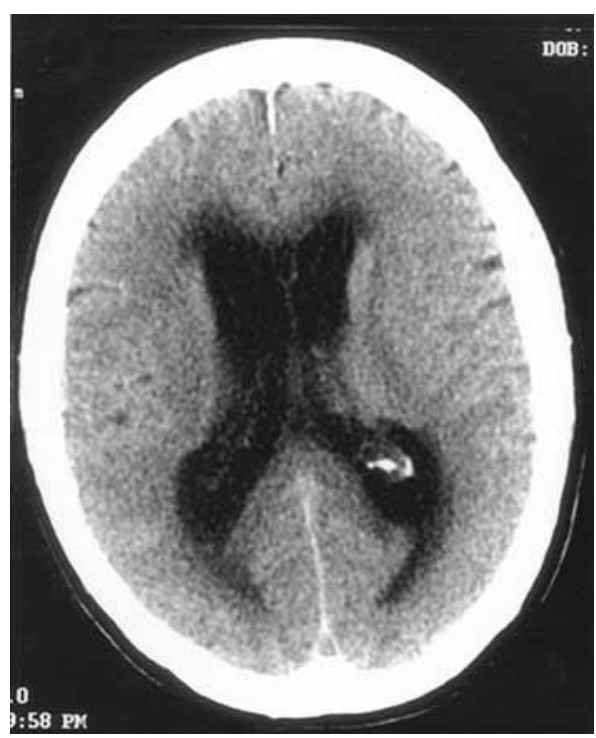

Figure 1. A, CT scan demonstrating a large, partly hemorrhagic infarct in the medial part of both cerebellar hemispheres. $B$, Cranial CT scan demonstrating moderate hydrocephalus with generalized sulcal effacement.

2. De Haan RJ, Limburg M, Van der Meulen JHP, Jacobs HM, Aaronson NK. Quality of life after stroke: impact of stroke type and lesion location. Stroke. 1995;26:402-8.

3. Koh MG, Phan TG, Atkinson JL, Wijdicks AF. Neuroimaging in deteriorating patients with cerebellar infarcts and mass effect. Stroke. 2000;31:2062-7.

4. Gonzalez-Santos JM, Gonzalez-Santos ML, Vallejo JL. Acute obstructive hydrocephalus: an unusual complication after cardiopulmonary bypass. Thorac Cardiovasc Surg. 1986;34:201-3.

5. Tyagi AK, Kirollos RW, Marks PV. Posttraumatic cerebellar infarction. Br J Neurosurg. 1995;9:683-6. 